\title{
THE GENERIC SOLUTION OF THE RIEMANN PROBLEM IN A NEIGHBORHOOD OF A POINT OF RESONANCE FOR SYSTEMS OF NONLINEAR BALANCE LAWS *
}

\author{
JOHN HONG ${ }^{\dagger}$ AND BLAKE TEMPLE
}

\begin{abstract}
We describe the generic solution of the Riemann problem near a point of resonance in a general $2 \times 2$ system of balance laws coupled to a stationary source. The source is treated as a conserved quantity in an augmented 3x3 system, and Resonance is between a nonlinear wave family and the stationary source. Transonic compressible Euler flow in a variable area duct, as well as spherically symmetric flow, are shown to be special cases of the general class of equations studied here.
\end{abstract}

1. Introduction. We consider a general $3 \times 3$ system of balance laws of the form

$$
\begin{aligned}
& a_{t}=0, \\
& w_{t}+f(a, w)_{x}=a^{\prime} g(a, w),
\end{aligned}
$$

where $(1.2)$ is a $2 \times 2$ system of conservation laws, $w=(u, v) \equiv\left(w_{1}, w_{2}\right), f=\left(f_{1}, f_{2}\right)$, $g=\left(g_{1}, g_{2}\right)$ and $(1.1)$ is incorporated to model resonance between a stationary source $a(x) \in \mathbf{R}$ and one of the nonlinear wave families of (1.2). Letting $U=(a, u, v)$, system (1.1), (1.2) is equivalent to the $3 \times 3$ system

$$
U_{t}+F(U)_{x}=a^{\prime} G(U)
$$

where $F=\left(0, f_{1}, f_{2}\right)$ and $G=\left(0, g_{1}, g_{2}\right)$.

Resonance occurs at states $U_{*}=\left(a_{*}, w_{*}\right)$ where an eigenvalue of $\frac{D f}{D w}(U)$ vanishes. A special case of system (1.1), (1.2) is given by the equations for compressible flow in a variable area duct,

$$
\begin{aligned}
& a_{t}=0, \\
& \rho_{t}+(\rho \mathrm{v})_{x}=-\frac{a^{\prime}}{a} \rho \mathrm{v}, \\
& (\rho \mathrm{v})_{t}+\left(\rho \mathrm{v}^{2}+p\right)_{x}=-\frac{a^{\prime}}{a} \rho \mathrm{v}^{2},
\end{aligned}
$$

where $\rho$ is the density, $p$ is the pressure, $\mathrm{v}$ is the velocity, and $a(x)$ is the diameter of the duct at position $x$. System (1.4)-(1.6) results under the assumption that $p$ is a function of $\rho$ alone, in which case the energy equation $(\rho E)_{t}+\left(\rho E_{\mathrm{V}}+p \mathrm{v}\right)_{x}=$ $-\frac{a^{\prime}}{a}(\rho E \mathrm{v}+p \mathrm{v})$ uncouples from the mass and momentum equations (1.5) and (1.6). Spherically symmetric $n$-dimensional flow arises when $-\frac{a^{\prime}}{a}=\frac{n-1}{x}$. System (1.4)-(1.6) is a $2 \times 2$ system with sources of form (1.1), (1.2), and the condition of resonance

\footnotetext{
*Received February 18, 2003; accepted for publication August 15, 2003.

${ }^{\dagger}$ Department of Mathematics, UCLA, Los Angeles, CA 90095-1555, USA (jhong@math.ucla.edu).

${ }^{\ddagger}$ Department of Mathematics, University of California, Davis, Davis CA 95616, USA (jbtemple@ucdavis.edu). Supported in part by NSF Applied Mathematics Grant Number DMS010-2493 and by the Institute of Theoretical Dynamics, UC-Davis.
} 
for system (1.4)-(1.6) translates into the statement that the flow is transonic in a neighborhood of $U_{*},[2]$.

Here we identify natural generic conditions on $f$ and $g$ at a point of resonance $U_{*}$ in system (1.1), (1.2) that guarantee a canonical solution of the Riemann problem in a neighborhood of $U_{*}$, and we then present the solution. As a special case we show that these conditions are met by system (1.4)-(1.6) - however, with a different choice of signs, qualitatively different solutions are possible. By identifying the generic local structure of the Riemann problem near a point of resonance, we accomplish a preliminary step in our program to extend the results in [7] to systems; that is, our program is to obtain a time independent estimate for the total variation of the conserved quantities near a point of resonance by analyzing approximate Glimm scheme solutions of system (1.3).

Note that because of the presence of the $a^{\prime}$ term in (1.2), the Riemann problem, (the initial value problem when the data is given by piecewise constant states), appears to be singular when $a$ is discontinuous. However, because of a re-scaling property of standing waves for systems of form (1.2), the Riemann problem really does give the elementary waves that provide the building blocks for more general solutions. For example, in [6] it was shown that Glimm's analysis of wave interactions and the local total variation bound extend to these singular Riemann problems for $n \times$ $n$ strictly hyperbolic systems of form (1.2), and convergence of the Glimm Scheme is proven for Lipschitz continuous $a$, (the case when the weak formulation of (1.2) applies). Interestingly, the residual converges weakly, by oscillation, rather than by $L^{1}$ convergence as in Glimm's original paper. These results were extended to resonant scalar balance laws in [7].

2. Generic Conditions. We discuss generic conditions on the functions $f(U)$, $g(U)$ for system (1.1), (1.2), that guarantee a canonical solution of the Riemann problem near a point of resonance $U_{*}$. This is preparatory to the presentation of the solution of the Riemann problem in the next section.

To start, let $\lambda_{i} \equiv \lambda_{i}(a, w)$ denote the eigenvalues, and $R_{i} \equiv R_{i}(a, w), L_{i} \equiv$ $L_{i}(a, w)$ corresponding right and left eigenvectors, respectively, for the $2 \times 2$ matrix $\frac{D f}{D w} \equiv\left(\frac{\partial f_{i}}{\partial w_{j}}\right)$ at fixed $a, i, j=1,2$. Let $\frac{D g}{D w} \equiv\left(\frac{\partial g_{i}}{\partial w_{j}}\right)$, the differential holding $a$ constant. (We will normalize $R_{i}, L_{i}$ below, but the conditions to follow are independent of normalization). We assume the following four generic conditions hold at the state $U_{*}$ :

$$
0=\lambda_{1}\left(U_{*}\right)<\lambda_{2}\left(U_{*}\right)
$$

$$
\left.\nabla \lambda_{i} \cdot R_{i}\right|_{U=U_{*}} \neq 0, i=1,2
$$

$$
\left.L_{i} \cdot\left(g-f_{a}\right)\right|_{U=U_{*}} \neq 0, i=1,2,
$$$$
\operatorname{Det}\left[\begin{array}{ll}
L_{1} \cdot\left(g-f_{a}\right), & L_{1} \cdot \frac{D g}{D w} \cdot R_{1} \\
L_{2} \cdot\left(g-f_{a}\right), & L_{2} \cdot \frac{D g}{D w} \cdot R_{1} .
\end{array}\right] \neq 0 .
$$ 
Our main result can be stated as follows:

THEOREM 1. Assume that system (1.1), (1.2) satisfies (2.1)-(2.4) at the state $U=U_{*}$. Then the solution of the Riemann problem has a unique canonical structure in a neighborhood of the state $U_{*}$, this being explicitly given in the Figures below.

Before we construct the solution of the Riemann problem for system (1.1), (1.2) in a neighborhood of $U_{*}$, under assumptions (2.1)-(2.3), we first verify (2.1)-(2.4) for the gas dynamics example (1.5), (1.6) when $U=(a, u, v) \equiv(a, \rho, \rho \mathrm{v}), f(U)=\left(\rho \mathrm{v}, \rho \mathrm{v}^{2}+p\right)$, and $g(U)=\left(-\frac{\rho \mathrm{v}}{a},-\frac{\rho \mathrm{v}^{2}}{a}\right)$. The eigenvalues and right eigenvectors for system $(1.5),(1.6)$ at fixed $a$ are given by,

$$
\begin{gathered}
\lambda_{1}=\mathrm{v}-\sigma, \quad \lambda_{2}=\mathrm{v}+\sigma, \\
R_{1}=-\left(1, \lambda_{1}\right)^{t r}, \quad R_{2}=\left(1, \lambda_{2}\right)^{t r},
\end{gathered}
$$

where the sound speed $\sigma$ is given by

$$
\sigma=\sqrt{p^{\prime}(\rho)}
$$

Since the left eigenvectors satisfy $L_{i} \cdot R_{j}=0, i \neq j$, we can choose

$$
L_{1}=\frac{1}{\rho}\left(-\lambda_{2}, 1\right), \quad L_{2}=\frac{1}{\rho}\left(-\lambda_{1}, 1\right) .
$$

The coordinate system of Riemann invariants $(r, s)$ can be defined in terms of $L_{1}$ and $L_{2}$ by

$$
\begin{aligned}
& \nabla r=L_{1}, \\
& \nabla s=L_{2},
\end{aligned}
$$

which yields

$$
\begin{aligned}
& r=v-\int_{\rho} \frac{\sqrt{p^{\prime}}}{\rho} \\
& s=v+\int_{\rho} \frac{\sqrt{p^{\prime}}}{\rho} .
\end{aligned}
$$

From (2.5)-(2.7) it follows that assumptions (2.1)-(2.4) respectively translate into the following conditions at $U_{*}=\left(a_{*}, u_{*}, v_{*}\right)$, (we assume equation of state $p=p(\rho)$, $\left.p^{\prime}(\rho)>0, p^{\prime \prime}(\rho)>0\right)$ :

$$
u_{*}=\sigma_{*}=\sqrt{p^{\prime}\left(\rho_{*}\right)}
$$

$$
\nabla \lambda_{i} \cdot R_{i}=\left\{\frac{p^{\prime \prime}}{2 \sigma}+\frac{\sigma}{\rho}\right\}>0, i=1,2
$$




$$
\begin{aligned}
& L_{1} \cdot\left(g-f_{a}\right)=\frac{1}{\rho}\left(-\lambda_{2}, 1\right) \cdot\left(\frac{-\rho \mathrm{v}}{a},-\frac{\rho \mathrm{v}^{2}}{a}\right)=\frac{\mathrm{v} \sigma}{a}>0, \\
& L_{2} \cdot\left(g-f_{a}\right)=\frac{1}{\rho}\left(-\lambda_{1}, 1\right) \cdot\left(\frac{-\rho \mathrm{v}}{a},-\frac{\rho \mathrm{v}^{2}}{a}\right)=-\frac{\mathrm{v} \sigma}{a}<0 .
\end{aligned}
$$

Finally, to verify (2.4), write

$$
\begin{aligned}
& L_{1} \cdot \frac{D g}{D w}=\frac{1}{\rho}\left(-\lambda_{2}, 1\right) \cdot\left[\begin{array}{cc}
0 & -\frac{1}{a} \\
\frac{v^{2}}{a} & -\frac{2 v}{a}
\end{array}\right]=\frac{1}{a \rho}\left(v^{2}, \lambda_{2}-2 v\right), \\
& L_{2} \cdot \frac{D g}{D w}=\frac{1}{\rho}\left(-\lambda_{1}, 1\right) \cdot\left[\begin{array}{cc}
0 & -\frac{1}{a} \\
\frac{v^{2}}{a} & -\frac{2 v}{a}
\end{array}\right]=\frac{1}{a \rho}\left(v^{2}, \lambda_{1}-2 v\right) .
\end{aligned}
$$

It follows that at $U=U_{*}, \lambda_{1}=0$, and so we have

$$
\begin{aligned}
& L_{1} \cdot \frac{D g}{D w} \cdot R_{1}=\frac{1}{a \rho}\left(-v^{2}+\lambda_{1}^{2}\right)=-\frac{v^{2}}{a \rho}<0, \\
& L_{2} \cdot \frac{D g}{D w} \cdot R_{1}=\frac{1}{a \rho}\left(-v^{2}+\lambda_{1} \lambda_{2}\right)=-\frac{v^{2}}{a \rho}<0 .
\end{aligned}
$$

The condition (2.4) now follows from (2.13), (2.14) and (2.15), (2.18).

3. The Riemann Problem. The Riemann problem is the initial value problem with initial data given at $t=0$ by the jump discontinuity

$$
U_{0}(x)= \begin{cases}U_{L}=\left(a_{L}, u_{L}\right) & \text { if } x<0, \\ U_{R}=\left(a_{R}, u_{R}\right) & \text { if } x>0 .\end{cases}
$$

We derive the solution of (3.1) for system (1.1), (1.2) within the class of elementary waves, shock waves, rarefaction waves and standing waves. It is easiest to display the solution in a coordinate system of Riemann invariants, where, with convenient conventions for $R_{i}, L_{i}$, the solution has a unique local structure.

At each fixed $a$, system (1.2) is a $2 \times 2$ system of conservation laws, and thus, since the state space is the plane, there exists a coordinate system of Riemann invariants $(r, s)$. Choose $(r, s)$ such that $L_{1}=\nabla r \equiv\left(\frac{\partial r}{\partial u}, \frac{\partial r}{\partial v}\right)$, and similarly, $L_{2}=\nabla s$. Also, let $\mathcal{T} \equiv\left\{U: \lambda_{1}(U)=0\right\}$ denote the transition surface. This is the surface where the speeds of 1-rarefaction waves change their sign relative to the local speed of standing waves, c.f. [8]. Since $\nabla \lambda_{1} \neq 0$, it follows that $\mathcal{T}$ is a smooth one dimensional surface passing through the base point $U=U_{*}$ and transversal to $R_{1}$ at that point, and hence the one wave curves cut the transition surface transversally in a neighborhood of $U_{*}$.

For convenience, choose the signs in (2.1)-(2.3) so that they agree with the signs in the gas dynamics example (2.11)-(2.13). That is, assume

$$
\nabla \lambda_{1} \cdot R_{1}>0
$$

$$
\left.L_{1} \cdot\left(g-f_{a}\right)\right|_{U=U_{*}}>0,
$$




$$
\left.L_{2} \cdot\left(g-f_{a}\right)\right|_{U=U_{*}}<0 .
$$

Under these assumptions, the solution of the Riemann problem is diagrammed in the figures below. Before discussing the solution of the Riemann problem, we first show that there is no loss of generality in the choice of signs (3.2)-(3.4), and discuss the significance of the sign in (2.4).

To start, note that we can fix the sign in (3.2) by choosing the orientation of $R_{1}$. We next show that the signs in (3.3) and (3.4) fix the convexity of standing wave curves relative to the $r$ and $s$ coordinate axes, and the sign in (2.4) then determines whether the zero speed shock curve, (c.f. [7]), lies inside or outside the corresponding standing wave curve, both of which eminate from the same point on the transition surface. More precisely, we show that (3.3) fixes the sign of $\frac{d^{2} a}{d r^{2}}$, and then (3.4) fixes the sign of $\frac{d^{2} s}{d r^{2}}$ at the point where a standing wave curve $U_{s}(r)$ crosses the transition surface $\lambda_{1}=0$. Then, with the choice of signs in (3.2)-(3.4), we show that the negative, [respectively positive], sign in (2.4) implies that zero speed shock curves, which emanate from points on the transition surface, break to the inside, [respectively outside], of the corresponding standing wave curves that emanates from the same point on the transition surface, in a neighborhood of $U=U_{*}$, c.f. [7].

To this end, let $U_{s}(x) \equiv\left(a_{s}(x), w_{s}(x)\right)$ denote a standing wave curve, obtained from (1.3) by assuming no temporal dependence. That is, $U_{s}$ satisfies

$$
\frac{D f}{D w} \cdot d w=\left(g-f_{a}\right) d a .
$$

Multiplying by $L_{i}$ on the left, $i=1,2$, we obtain

$$
\begin{aligned}
& \lambda_{1} L_{1} \cdot d w=L_{1} \cdot\left(g-f_{a}\right) d a, \\
& \lambda_{2} L_{2} \cdot d w=L_{2} \cdot\left(g-f_{a}\right) d a .
\end{aligned}
$$

Using $L_{1} \cdot d w=d r, L_{2} \cdot d w=d s$, we obtain

$$
\begin{aligned}
& \lambda_{1} d r=L_{1} \cdot\left(g-f_{a}\right) d a, \\
& \lambda_{2} d s=L_{2} \cdot\left(g-f_{a}\right) d a,
\end{aligned}
$$

which leads to

$$
\begin{aligned}
\frac{d a}{d r} & =\frac{\lambda_{1}}{L_{1} \cdot\left(g-f_{a}\right)}, \\
\frac{d s}{d r} & =\frac{\lambda_{1} \cdot\left\{L_{2} \cdot\left(g-f_{a}\right)\right\}}{\lambda_{2} \cdot\left\{L_{1} \cdot\left(g-f_{a}\right)\right\}} .
\end{aligned}
$$

Note that (3.10) implies that standing wave curves are tangent to 1-wave curves at the transition surface $\lambda_{1}=0$. We now show that (3.4) implies that this tangency is quadratic. (Since shock wave curves have cubic tangency with rarefaction curves, this implies that shock curves emanating from left states on $U_{s}$ and crossing into 
the convex side of the standing wave curve, must cross the standing wave curve at a unique point on the opposite side of $\mathcal{T}$ ). So consider the standing wave curve at a point on the transition surface $\mathcal{T}$. Then (3.10), (3.11) and (3.3) imply that

$$
\begin{aligned}
\frac{d^{2} a}{d r^{2}} & =\frac{1}{L_{1} \cdot\left(g-f_{a}\right)} \frac{d \lambda_{1}}{d r}>0, \\
\frac{d^{2} s}{d r^{2}} & =\frac{L_{2} \cdot\left(g-f_{a}\right)}{\lambda_{2} L_{1} \cdot\left(g-f_{a}\right)} \frac{d \lambda_{1}}{d r}<0,
\end{aligned}
$$

gives the curvature in $a$ and $s$ of the standing wave curve at a point on the transition surface. By (3.12), we have shown that (3.3) determines the sign of $\frac{d^{2} a}{d r^{2}}$, and (3.4) determines the sign of $\frac{d^{2} s}{d r^{2}}$ along a standing wave curve at the point where it crosses the transition surface, as claimed.

We can now show that (2.4) determines whether the zero speed shock curves break to the left or to the right of the standing wave curve at the point on the transition surface at which they emanate. To define the zero speed shock curve, start with a fixed standing wave curve $U_{s}(r)$. This curve crosses the transition surface at a unique point in a neighborhood of $U_{*}$, and so as not to introduce more notation, assume without loss of generality that this point is $U_{*}$ itself. By our sign conventions, the wave speed $\lambda_{1}$ increases moving to the right through the transition surface, (that is, toward increasing $r$ ), along the standing wave curve $U_{s}(r)$ in the $(r, s)$-plane. Consider the portion of a standing wave curve that lies to the right of the transition surface; that is, consider $U_{s}(r)=\left(a_{s}(r), u_{s}(r), v_{s}(r)\right)$ for $r>r_{*}$, where $U_{s}\left(r_{*}\right)=U_{*} \in \mathcal{T}$. Then $\lambda_{1}$ increases from $\lambda_{1}\left(U_{*}\right)=0$ as $r$ increases from $r=r_{*}$ along $U_{s}(r)$, and thus it follows that for each $r>r_{*}$, there is a unique state $\tilde{U}_{s}(r)$ on the left of the transition surface such that the shock $\left[U_{s}(r), \tilde{U}_{s}(r)\right]$ is a zero speed shock when the left state of the shock is $U_{s}(r)$ and the right state is $\tilde{U}_{s}(r)$. We call the curve $\tilde{U}_{s}(r)$ the zero speed shock curve associated with the standing wave curve $U_{s}(r)$. (This is diagrammed in Figures 1 and 2, in the cases when the standing wave curve lies to the left and right of the zero speed shock curve, respectively, assuming normalization (3.2)-(3.4). The dotted line denotes the zero speed shock curve, and its associated standing wave curve is drawn as the parabolic curve through state $U-U_{*}$. Our convention is that the zero speed shock curve is parameterized by values of $r>r_{*}$ that parameterize $U_{s}$ on the right of $\mathcal{T}$, and observe that $U_{s}(r)$ and $\tilde{U}_{s}(r)$ are curves that lie at the same value of $a$. As a parameterized curve, $\tilde{U}_{s}(r)$ lies to the left of $\mathcal{T}$, and emanates from the state $U=U_{*}$, where it is tangent to $R_{1}$.) Note that since shock curves have third order tangency with $s=$ const at the state $U_{L}$, and the standing wave curves have quadratic tangency, it follows that the shock curves emanating from left states on $U_{s}(r)$ for $r>r_{*}$, always break transversally into the region below the standing wave curve, and hence, in a sufficiently small neighborhood, such shock curves intersect the standing wave curve $U_{s}$ at exactly two points: at the left state $U_{L}=U_{s}(r)$ on the right of $\mathcal{T}$, and at a unique point $U_{R}$ on the left of $\mathcal{T}$. We now show that, (with the choice of signs in (3.2)-(3.4)), the point $\tilde{U}_{s}(r)$ on the zero speed shock curve always lies on the shock curve emanating from $U_{L}$ between $U_{L}$ and $U_{R}$ when the sign in (2.4) is negative, (that is, the shock wave $\left[U_{L}, U_{R}\right]$ has negative speed), while the point $U_{R}$ lies on the shock curve emanating from $U_{L}$ between $U_{L}$ and $\tilde{U}_{s}(r)$ when the sign in (2.4) is positive, (so that the shock wave $\left[U_{L}, U_{R}\right]$ has positive speed in this case). To this end, consider the zero speed shock $\left[U_{s}\left(r_{s}\right), \tilde{U}_{s}\left(r_{s}\right)\right], r_{s}>r_{*}$. (We use $r_{s}$ in this argument to indicate the $r$-parameterization of $U_{s}$ on the right of $\mathcal{T}$.) 
Since the shock has zero speed, the Rankine-Hugoniot jump conditions imply that $f\left(U_{s}\left(r_{s}\right)\right)=f\left(\tilde{U}_{s}\left(r_{s}\right)\right)$, and $a_{s}\left(r_{s}\right)=\tilde{a}_{s}\left(r_{s}\right)$, so we can write

$$
f\left(a_{s}\left(r_{s}\right), \tilde{u}_{s}\left(r_{s}\right), \tilde{v}_{s}\left(r_{s}\right)\right)=f\left(a_{s}\left(r_{s}\right), u_{s}\left(r_{s}\right), v_{s}\left(r_{s}\right)\right) .
$$

Using the condition for a standing wave $d f=g d a$, we can express the increments $d f$ on the left and right sides of (3.14), corresponding to an increment $d r_{s}$, by

$$
d f=f_{a}\left(\tilde{U}_{s}\right) d a_{s}+\frac{D}{D w} f\left(\tilde{U}_{s}\right) d \tilde{w}_{s}=g\left(U_{s}\right) d a_{s}
$$

which leads to

$$
\frac{D}{D w} f\left(\tilde{U}_{s}\right) d \tilde{w}_{s}=\left[g\left(\tilde{U}_{s}\right)-f_{a}\left(\tilde{U}_{s}\right)\right] d a_{s}+\left[g\left(U_{s}\right)-g\left(\tilde{U}_{s}\right)\right] d a_{s}
$$

But

$$
\left[g\left(U_{s}\right)-g\left(\tilde{U}_{s}\right)\right]=\frac{D}{D w} g\left(\tilde{U}_{s}\right) \cdot\left(W_{s}-\tilde{W}_{s}\right)+O\left(\left|W_{s}-\tilde{W}_{s}\right|^{2}\right)
$$

and

$$
W_{s}-\tilde{W}_{s}=\epsilon R_{1}\left(\tilde{U}_{s}\right)+O\left(\left|W_{s}-\tilde{W}_{s}\right|^{2}\right)
$$

where

$$
\epsilon=O\left(\left|W_{s}-\tilde{W}_{s}\right|\right)
$$

Multiplying (3.16) by $L_{i}, i=1,2$, using $L_{1} \cdot \frac{D f}{D w}=\lambda_{1} \cdot L_{1}=\lambda_{1} \nabla r, L_{2} \cdot \frac{D f}{D w}=\lambda_{2} \cdot L_{2}=$ $\lambda_{2} \nabla s$, and using (3.17) and (3.18), we obtain the derivatives of $a$ and $s$ with respect to the value of $r$ on the zero speed shock curve to the left of $\mathcal{T}$,

$$
\begin{aligned}
& \frac{d a}{d r}=\frac{\lambda_{1}}{L_{1} \cdot\left(g-f_{a}\right) d a+\epsilon\left(L_{1} \cdot \frac{D g}{D w} \cdot R_{1}\right)}+O\left(\epsilon^{2}\right), \\
& \frac{d s}{d r}=\frac{\lambda_{1}}{\lambda_{2}} \frac{L_{2} \cdot\left(g-f_{a}\right)+\epsilon\left(L_{2} \cdot \frac{D g}{D w} \cdot R_{1}\right)}{L_{1} \cdot\left(g-f_{a}\right)+\epsilon\left(L_{1} \cdot \frac{D g}{D w} \cdot R_{1}\right)}+O\left(\epsilon^{2}\right) .
\end{aligned}
$$

Note that setting $\epsilon=0$ in (3.20), (3.21) gives the formulas (3.10), (3.11) for the corresponding derivatives of the standing wave curve at $U=U_{*}$. Furthermore, taking the derivative of (3.21) with respect to $\epsilon$ at $\epsilon=0$, we see that the zero speed shock curve will break transversally to one side or the other of the standing wave curve at $U=U_{*}$ if the determinant in (2.4) is non-zero. Using the choice of signs in (3.2)(3.4), it follows that $\tilde{U}_{s}(r)$ lies to the right, [respectively left], of $U_{s}(r)$ for $r<r_{*}$ if the determinant in (2.4) is positive, [respectively negative], (diagrammed in Figures 1 and 2 , respectively), as claimed. 
The solution of the Riemann problem is diagrammed in Figures 3-10, assuming the normalization (3.2)-(3.4). As an entropy condition we take the condition that the change in $a$ is monotone along standing waves in a solution. This allows for what we call triple composite standing waves, and also entails a non-uniqueness of solutions, and a corresponding lack of continuous dependence of the solutions on $U_{L}$ and $U_{R}$. This was observed in the scalar case in $[14,7]$, and represents an interesting complication in the structure of the possible time asymptotic wave patterns. Even so, the analysis here shows that this interesting structure is canonical in a neighborhood of a state of resonance. The analysis of the Glimm scheme for the scalar case treated in $[14,7]$ leads to additional entropy conditions that further restrict the admissible solution of the Riemann problem. In particular, the $L_{w}$ minimization principle introduced in [7], determined a unique solution of the Riemann problem, except for an inherent duplicity of solutions at boundaries where the qualitative wave structure of solutions changes. For our purposes here, we make no further entropy restrictions, c.f. [7].

Figures 3-6 give the solution of the Riemann problem in the case when the zero speed shock curve, (drawn as a dashed, downward parabolic curve to the left of $\mathcal{T}$ in each diagram), lies to the right of the standing wave curve, (the case of a positive sign in (2.4)), and Figures 7-10 give the case when the zero speed shock curve lies to the right of the standing wave curve, (the case of a negative sign in (2.4)), according to the four cases $a_{L}$ less than or greater than $a_{R}$, and $U_{L}$ left or right of $\mathcal{T}$. (The gas dynamics system (1.4)-(1.6) is of the type diagrammed in Figures 3-6.) To keep the diagrams as simple as possible, we make the following idealizations. First, the 1wave curves and 2-wave curves are drawn as horizontal and vertical lines, respectively. More specifically, the third order tangency of shock curves and rarefaction curves is neglected as a higher order effect relative to the quadratic tangency of standing wave curves and 1-wave curves in each diagram. Also, the states at level $a=a_{R}$ are drawn along a single horizontal line. In fact, the 1- and 2-wave curves lie at constant $a$, so the change in $a$ in a Riemann problem enters as a jump across the standing waves. Thus, the states at level $a=a_{R}$ would actually be a curve of states obtained by maintaining the condition $\Delta a=a_{R}-a_{L}$ along the standing wave in the solution. The value of $a$ changes quadratically along standing wave curves, and by our conventions, $a$ takes a minimum at the point of intersection of the standing wave with the transition surface.

The dark curves in Figures $3-10$ represent states that can be reached by a 1 -wave, or by 1 -waves and the critical standing wave that marks the place where the 1 -waves and standing waves change their relative speed. In each diagram, the solution is a combination of 1 -waves and standing waves, followed by a 2 -wave, where the order of 1-waves and standing waves is determined by taking the slower wave first. In particular, by (3.2)-(3.4), 1-waves with left state $U_{L}$ to the right of $\mathcal{T}$ have positive speed, (and hence come after the zero speed standing waves moving from left to right in the $x$-plane), unless the 1 -wave is a shock wave with a left state to the left of the zero speed shock curve.

In the cases of Figures 3-10 excluding the cases of Figures 5 and 9, the solution of the Riemann problem can contain what we call triple composite standing waves. This is a standing wave that consists of a standing wave to the right of $\mathcal{T}$, followed by a zero speed shock wave, followed by a different standing wave to the left of $\mathcal{T}$, where the sum of the changes in $a$ along the standing waves is $\Delta a=a_{R}-a_{L}$. Since all waves move with zero speed, a triple composite standing wave can be treated as a single wave. In each diagram, the region between the two vertical dashed lines, 
(which actually represent 2-wave curves), define the values of $U_{R}$ for which there is a solution $\left[U_{L}, U_{R}\right]$ that contains a triple composite standing waves. We now discuss the diagrams individually.

Discussion of Figure 3: The dashed line emanating from state $U_{*}$ is the zero speed shock wave. The corresponding standing wave curve is drawn to the right of $\mathcal{T}$ as the dark, downward parabolic curve emanating from the state $U_{*}$, continuing to the left of $\mathcal{T}$ as the solid parabolic curve emanating from $U_{*}$. To obtain the solution for given right state $U_{R}$ that lies to the right of state $D$ in the figure ${ }^{3}$, start at $U_{L}$, take the 1-wave curve, (the horizontal line through $U_{L}$ ), to an intermediate state left of $U_{*}$, follow this by the standing wave curve to the level $a=a_{R}$, (drawn as a horizontal line), and then take a two wave curve at level $a_{R}$ to $U_{R}$. When $U_{R}$ lies between the 2 -wave curves through $D$ and $E$, take the 1 -wave to $U_{*}$, (a rarefaction wave), take the triple composite standing wave to $C$, and then the two wave from $C$ to $U_{R}$; that is, the solution is $U_{L} \rightarrow U_{*} \rightarrow A \rightarrow B \rightarrow C \rightarrow U_{R}$. The triple composite standing wave is the standing wave from $U_{*}$ to $A$ followed by the zero speed shock from $A$ to $B$ followed by the standing wave from $B$ to $C$. Finally, if $U_{R}$ lies to the right of state $E$, take the rarefaction wave from $U_{L}$ to $U_{*}$, the critical standing wave from $U_{*}$ to level $a_{R}$, and then a positive speed 1-wave at level $a_{R}$ to a state right of $E$ that connects by a 2-wave to the state $U_{R}$. In this case the solution of the Riemann problem is unique.

Discussion of Figure 4: The solution is a negative speed 1-shock followed by a standing wave followed by a two wave when the state $U_{R}$ lies to the left of state $D$. When $U_{R}$ lies between $D$ and $E$, the solution consists of a triple composite wave to a state between $D$ and $E$, followed by a 2-wave to $U_{L}$, for example, $U_{L} \rightarrow A \rightarrow B \rightarrow$ $C \rightarrow U_{R}$, diagrammed in the figure. For $U_{R}$ to the right of state $E$, the solution consists of a standing wave followed by a positive speed 1-wave followed by a 2 -wave. In this case the solution of the Riemann problem is unique.

Discussion of Figure 5: The solution consists of a 1-wave followed by a standing wave followed by a 2 -wave. In this case the solution of the Riemann problem is unique.

Discussion of Figure 6 : The solution consists of a negative speed shock wave, (to a state left of $D$ ), followed by a standing wave for states $U_{R}$ which lie to the left of state $F$. However, states between $F$ and $G$ can also be solved by a triple composite standing wave followed by a 2 -wave, as in the solution $U_{L} \rightarrow A \rightarrow B \rightarrow C \rightarrow U_{R}$, or by a negative speed shock to a state left of $E$, followed by a standing wave, and then a 2 -wave. When $U_{R}$ lies to the right of state $G$, the solution consists of a standing wave followed by a positive speed 1 -wave followed by a 2 -wave. In this case there is a triple non-uniqueness of solutions when $U_{R}$ lies between states $F$ and $G$.

Discussion of Figure 7: For states $U_{R}$ to the left of state $D$, the solution consists of a negavive speed 1-wave followed by a standing wave to level $a=a_{R}$, followed by a 2-wave. For states $U_{R}$ between $D$ and $F$, the solution has a triple multiplicity: a 1 -wave followed by a standing wave followed by a 2-wave; or a 1-rarefaction wave to $U_{*}$ followed by the critical standing wave to $B$ followed by a positive speed 1 -wave to states between $D$ and $F$, followed by a 2 -wave to $U_{R}$; or else solutions with a triple composite standing wave like the solution $U_{L} \rightarrow U_{*} \rightarrow A \rightarrow C \rightarrow E \rightarrow U_{R}$. When $U_{R}$ lies to the right of state $F$, the solution reduces to the single solution $U_{L} \rightarrow U_{*} \rightarrow B$

\footnotetext{
${ }^{3}$ We say the state lies to the right of a state if it lies to the right of the 2 -wave curve through that state, drawn as a vertical line in the diagrams.
} 
followed by a positive speed 1-wave followed by a 2 -wave. In this case there is a triple multiplicity of solutions when $U_{R}$ lies between states $D$ and $F$.

Discussion of Figure 8: For states $U_{R}$ to the left of state $D$, the solution consists of a negative speed 1-wave followed by a standing wave to level $a=a_{R}$, followed by a 2-wave. For states $U_{R}$ between $D$ and $E$, the solution has a triple multiplicity: a negative speed 1-shock followed by a standing wave followed by a 2 -wave; or the critical standing wave from $U_{L}$ to $E$ followed by a positive speed 1-wave to a state between $D$ and $E$, followed by a 2 -wave to $U_{R}$; or else a solution with a triple composite standing wave like the solution $U_{L} \rightarrow A \rightarrow B \rightarrow C \rightarrow U_{R}$. When $U_{R}$ lies to the right of state $E$, the solution reduces to the single solution $U_{L} \rightarrow H$ followed by a positive speed 1 -wave followed by a 2 -wave. In this case there is a triple multiplicity of solutions when $U_{R}$ lies between states $D$ and $E$.

Discussion of Figure 9: This agrees with Figure 5.

Discussion of Figure 10: For states $U_{R}$ to the left of state $F$, the solution consists of a negavive speed 1-wave followed by a standing wave to level $a=a_{R}$, followed by a 2-wave. For states $U_{R}$ between $F$ and $G$, the solution consists of a triple composite standing wave to a state between $F$ and $G$, followed by a 2 -wave. And for right states $U_{R}$ to the right of $G$, the solution consists of a critical standing wave to level $a=a_{R}$, followed by a positive speed 1-wave, followed by a 2 -wave to $U_{R}$. In this case the solution of the Riemann problem is unique.

\section{REFERENCES}

[1] G. Chen And J. Glimm, Global solution to the compressible Euler equations with geometrical structure, Comm. Math. Phys., 179 (1996), pp. 153-193.

[2] R. Courant And K.O. Friedrichs, Supersonic flow and shock waves, John Wiley \& Sons, New York, 1948.

[3] G. Dal Maso, P.G. LeFloch, and F. Murat, Definition and weak stability of nonconservative products, J. Math. Pures Appl., 74 (1995), pp. 483-548.

[4] J. GLImM, Solutions in the large for nonlinear hyperbolic systems of equations, Comm. Pure Appl. Math., 18 (1965), pp. 697-715.

[5] S.K. Godunov, A difference method for numerical calculations of discontinuous solutions of the equations of hydrodynamics, Mat. Sb., 47 (1959), in Russion, pp. 271-306.

[6] J. Hong, The Glimm scheme extended to inhomogeneous systems, Doctoral Thesis, UC-Davis.

[7] J. Hong And B. Temple, A bound on the total variation of the conserved quantities for solutions of a general resonant nonlinear balance law, SIAM J. Appl. Math., to appear.

[8] E. ISAACSON, Global solution of a Riemann problem for a non-strictly hyperbolic system of conservation laws arising in enhanced oil recovery, Rockefeller University preprint.

[9] E. Isaacson, D. Marchesin, B. Plohr, and B. Temple, The Riemann problem near a hyperbolic singularity: the classification of solutions of quadratic Riemann problems I, SIAM J. Appl. Math., 48 (1988), pp. 1009-1052.

[10] E. IsaAcson, B. Temple, The structure of asymptotic states in a singular system of conservation laws, Adv. Appl. Math., 11 (1990), pp. 205-219.

[11] E. IsaAcson, B. Temple, Analysis of a singular hyperbolic system of conservation laws, Jour. Diff. Equn., 65 (1986), pp. 250-268.

[12] E. IsaAcson, B. Temple, Examples and classification of non-strictly hyperbolic systems of conservation laws, Abstracts of AMS, January 1985.

[13] E. IsaAcson, B. Temple, Nonlinear resonance in systems of conservation laws, with E. Isaacson, SIAM Jour. Appl. Anal., 52 (1992), pp. 1260-1278.

[14] E. IsaAcson, B. Temple, Convergence of the $2 \times 2$ Godunov method for a general resonant nonlinear balance law, SIAM Jour. Appl. Math., 55:3 (1995), pp. 625-640.

[15] B. Keyfitz AND H. KRANZER, A system of non-strictly hyperbolic conservation laws arising in elasticity theory, Arch. Rat. Mech. Anal., 72 (1980), pp. 219-241. 
[16] S.N. KRUZKov, First order quasilinear equations with several space variables, Mat. USSR Sb., 10 (1970), pp. 217-243.

[17] P.D. LAX, Hyperbolic systems of conservation laws, II, Comm. Pure Appl. Math., 10 (1957), pp. 537-566.

[18] P.D. Lax And B. Wendroff, Systems of Conservation laws, Comm. Pure Appl. Math., 13 (1960), pp. 217-237.

[19] L. Lin, J. Wang And B. Temple, A comparison of convergence rates for Godunov's method and Glimm's method in resonant nonlinear systems of conservation laws, with L.Lin and J. Wang., SIAM J. Numer. Anal., 32:3, pp. 824-840.

[20] L. Lin, J. WANG AND B. Temple, Suppression of oscillations in Godunov's method for a resonant non-strictly hyperbolic system, SIAM J. Numer. Anal., 32:3, June 1995.

[21] T.P. Liu, Quasilinear hyperbolic systems, Comm. Math. Phys., 68 (1979), pp. 141-172.

[22] T.P. LiU, Resonance for a quasilinear hyperbolic equation, J. Math. Phys., 28:11 (1987), pp. $2593-2602$.

[23] D. Marchesin and P.J. Paes-Leme, A Riemann problem in gas dynamics with bifurcation, Computers and Mathematics with Applications, 12A (1986), pp. 433-455.

[24] O.A. OLEINIK, Discontinuous solutions of non-linear differential equations, Uspekhi Mat. Nauk (N.S.), 12 (1957), no.3 (75), pp. 3-73 (Am. Math. Soc. Trans., Ser. 2, 26, pp. 195-172).

[25] J. SMoller, Shock waves and reaction diffusion equations, Springer-Verlag, Berlin, New York, 1983.

[26] B. Temple, Global solution of the Cauchy problem for a class of $2 \times 2$ nonstrictly hyperbolic conservation laws, Adv. in Appl. Math., 3 (1982), pp. 335-375.

[27] A. Tveito And R. Winther, Existence, uniqueness and continuous dependence for a system of hyperbolic conservation laws modelling polymer flooding, Preprint, Department of Informatics, University of Oslo, Norway, January, 1990. 


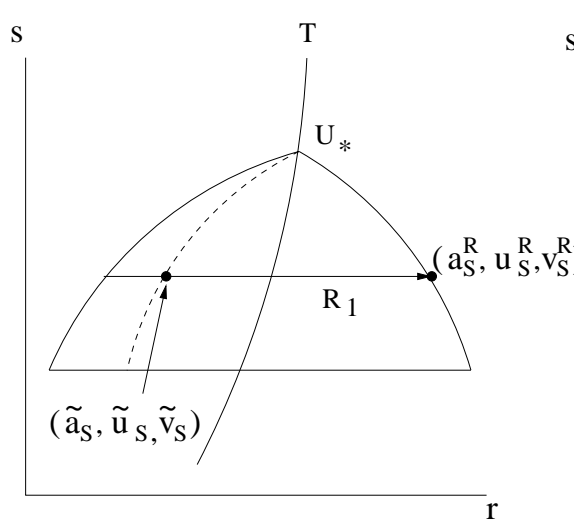

Figure 1

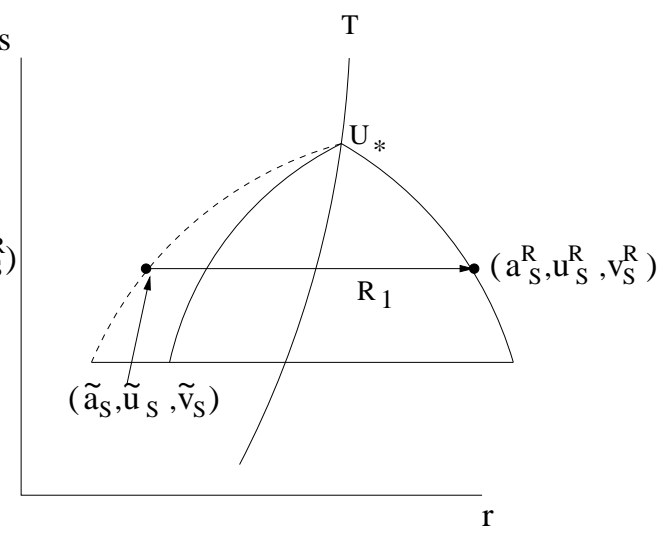

Figure 2

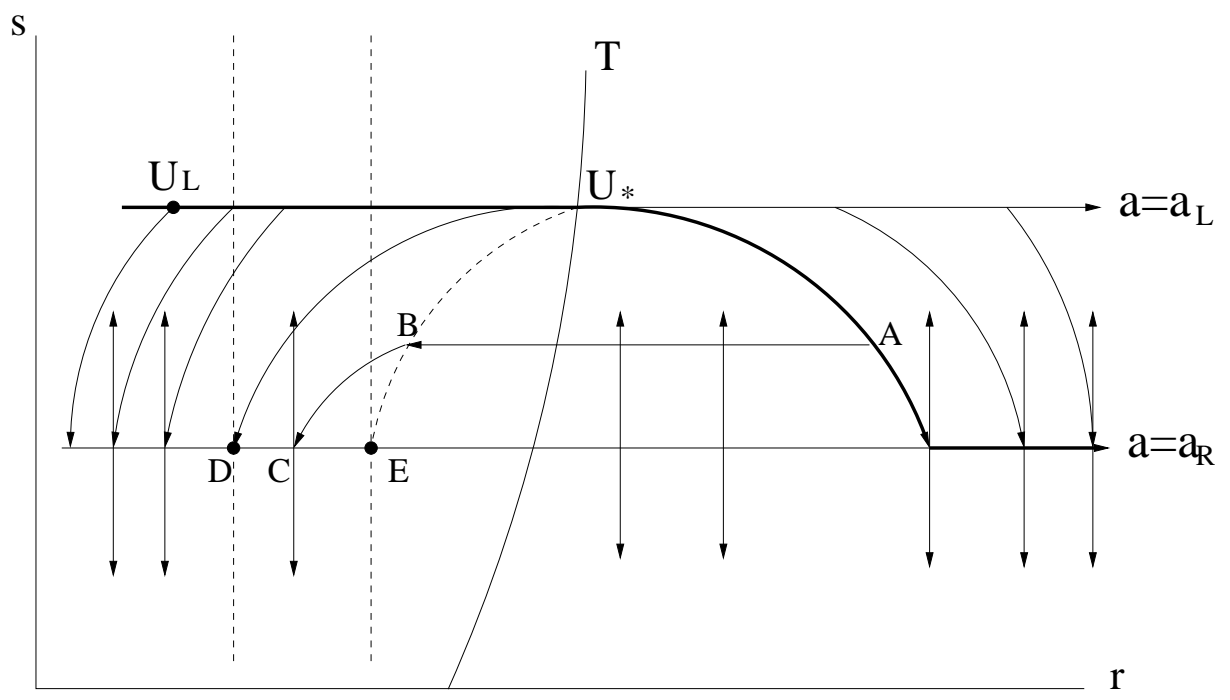

Figure $3 \quad\left(a_{R}>a_{L}, U_{L}<T\right)$ 


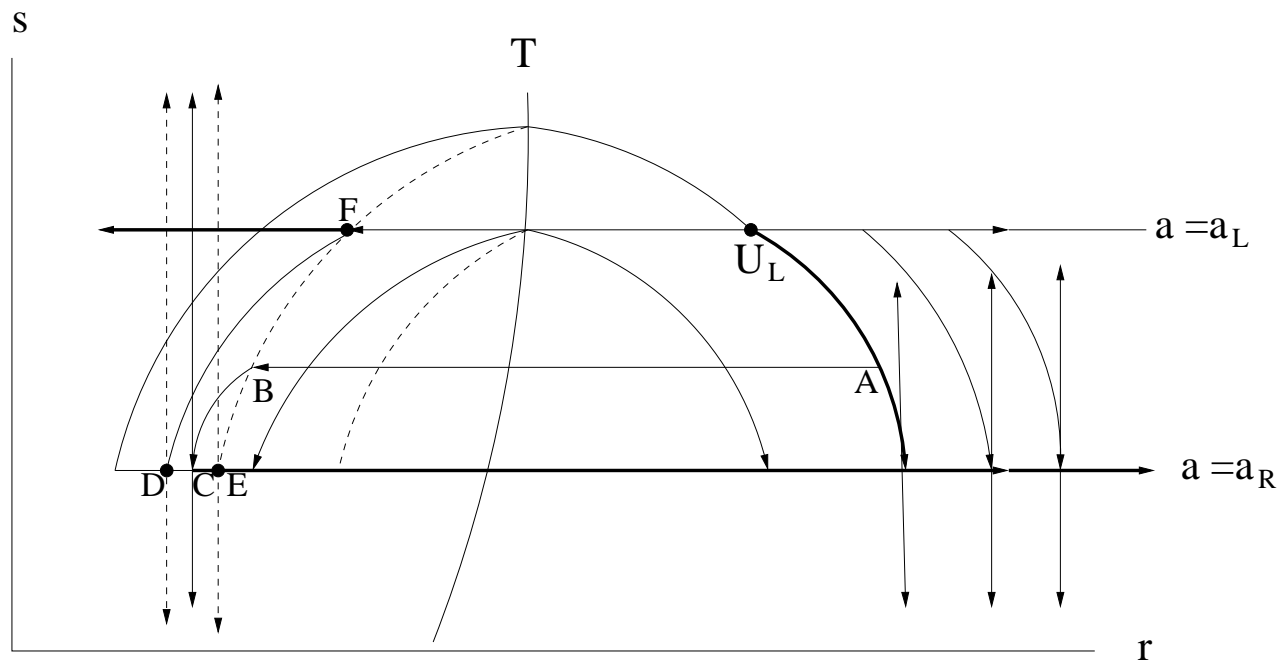

Figure $4 \quad\left(a_{R}>a_{L}, U_{L}>T\right)$

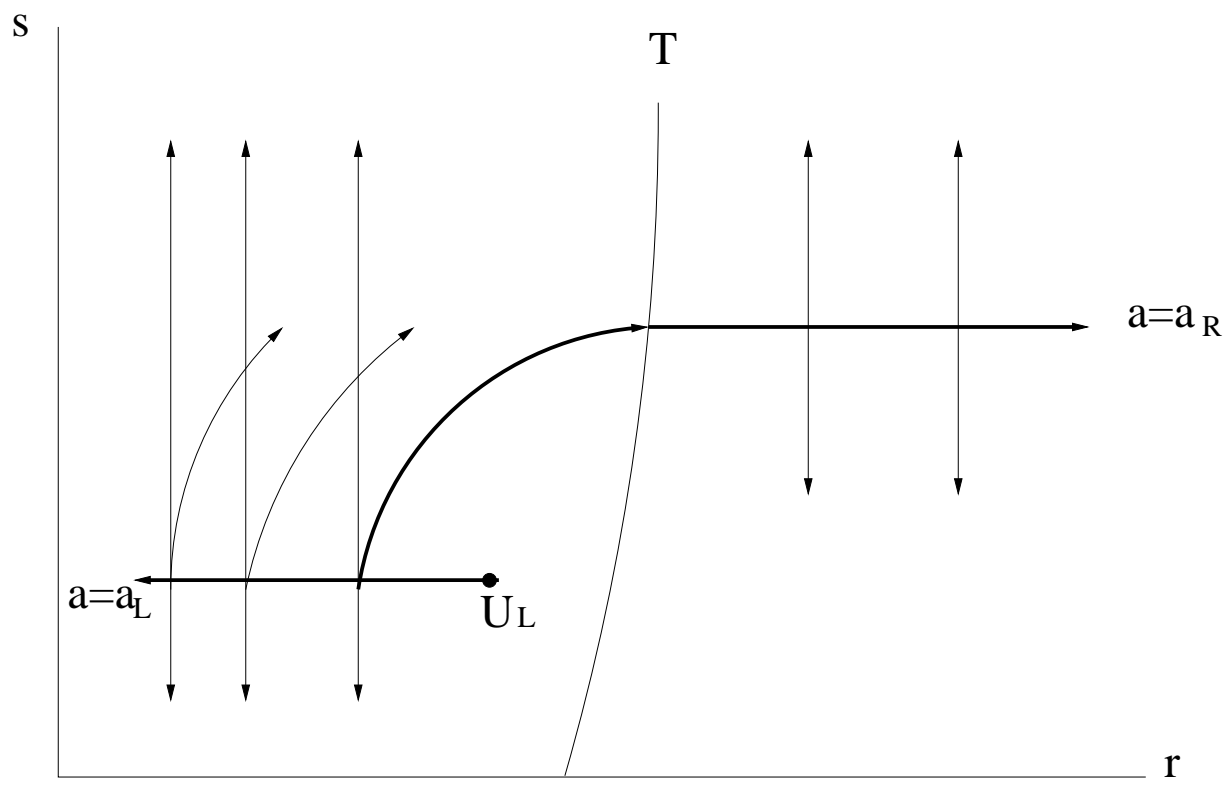

Figure $5 \quad\left(a_{R}<a_{L}, U_{L}<T\right)$ 


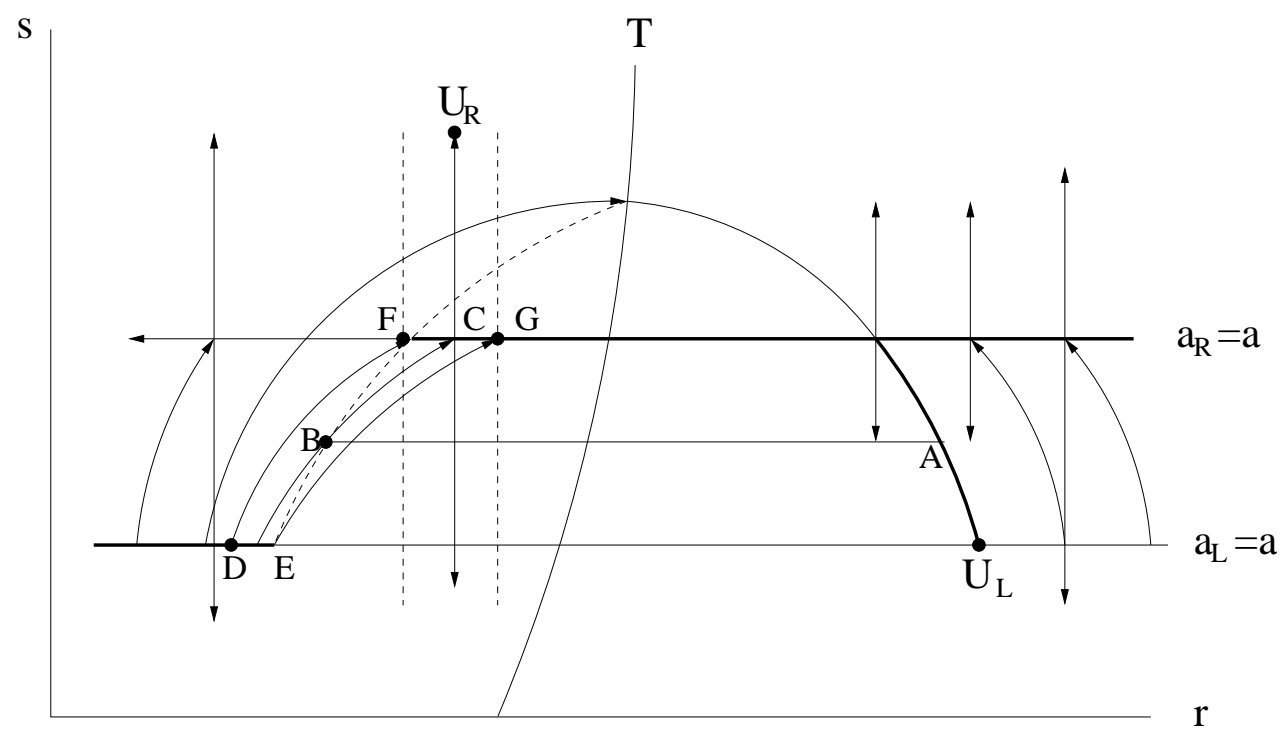

Figure $6 \quad\left(a_{R}<a_{L}, U_{L}>T\right)$

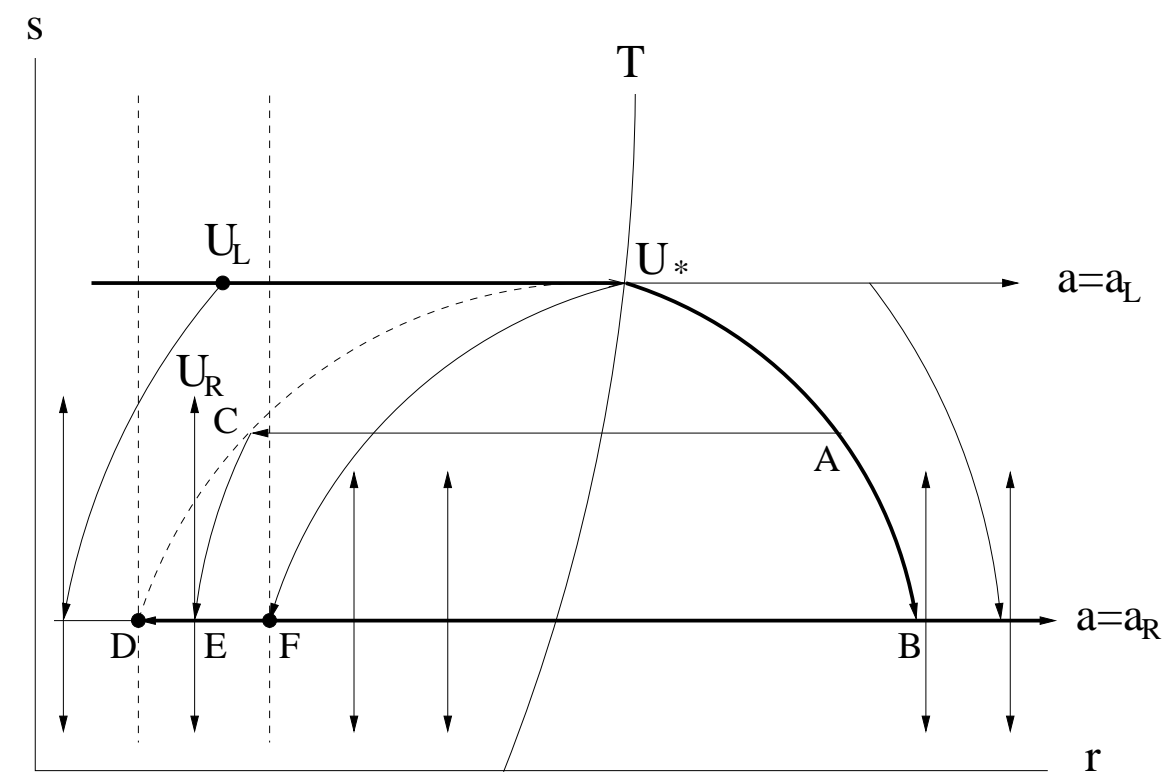

Figure $7 \quad\left(a_{R}>a_{L}, U_{L}<T\right)$ 


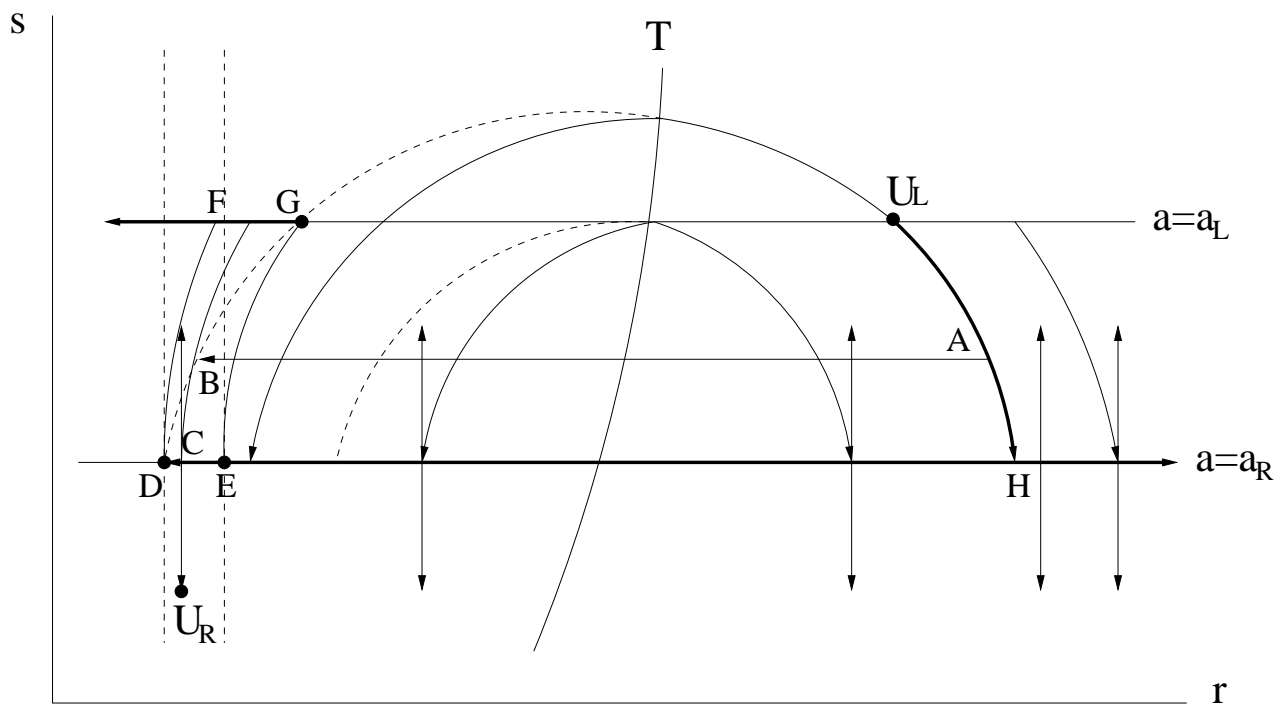

Figure $8 \quad\left(a_{R}>a_{L}, U_{L}>T\right)$

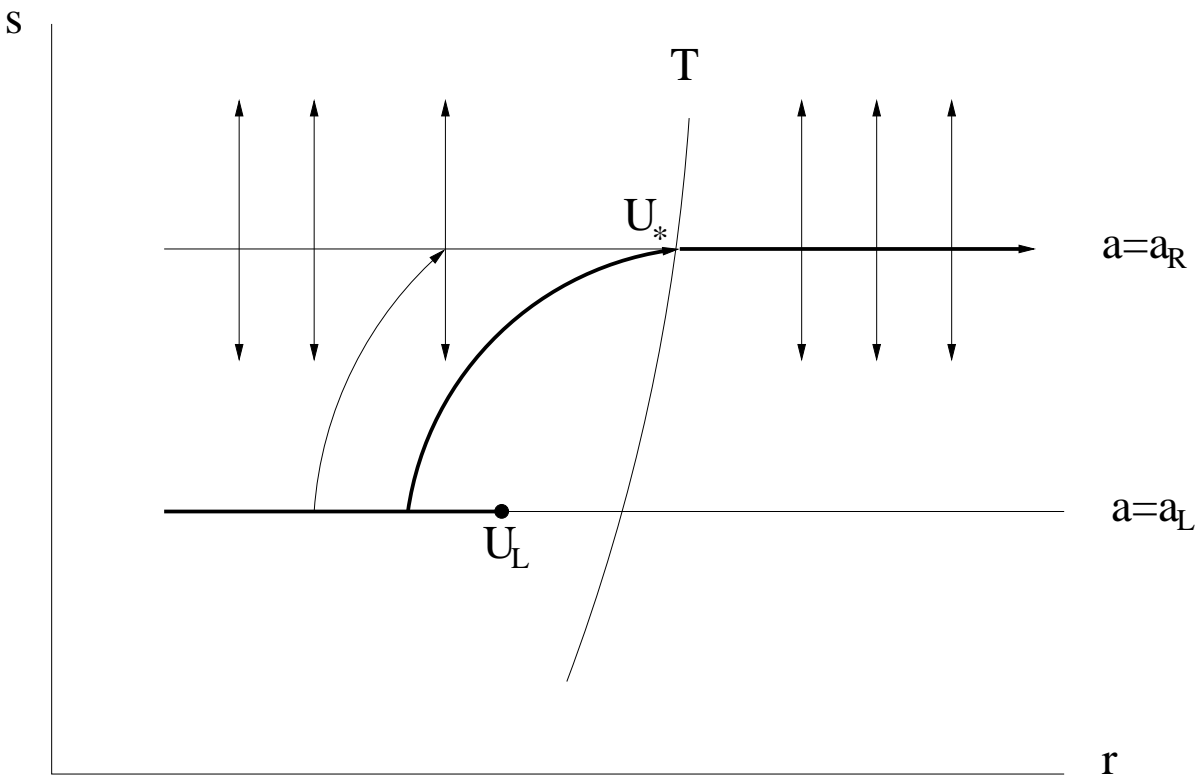

Figure $9 \quad\left(\mathrm{a}_{\mathrm{R}}<\mathrm{a}_{\mathrm{L}}, \mathrm{U}_{\mathrm{L}}<\mathrm{T}\right)$ 


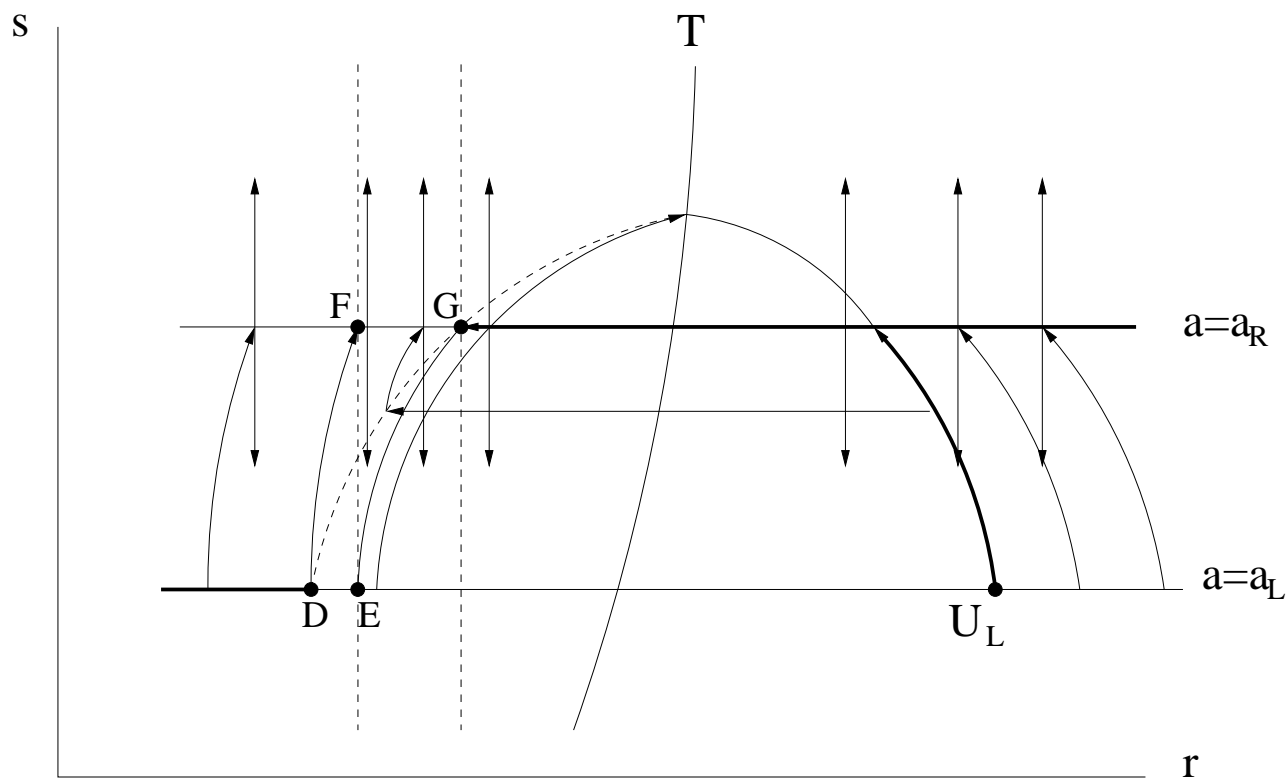

Figure $10\left(\mathrm{a}_{\mathrm{R}}<\mathrm{a}_{\mathrm{L}}, \mathrm{L}_{\mathrm{L}}>\mathrm{T}\right)$ 